FTUV-IFIC 99-11

\title{
Operator approach to the Gluing Theorem in String Field Theory
}

\author{
Abdulmajeed ABDURRAHMAN \\ abdurrahman@v2.rl.ac.uk \\ Rutherford Appleton Laboratory, \\ Chilton, Didcot, Oxon OX11 0QX, United Kingdom \\ José BORDES円 \\ jose.m.bordes@ uv.es \\ Dept. Fisica Teorica, Univ. de Valencia, \\ c. Dr. Moliner 50, E-46100 Burjassot (Valencia), Spain \\ and \\ Cristóbal LARA \\ Dept. Fisica Teorica, Univ. de Valencia, \\ c. Dr. Moliner 50, E-46100 Burjassot (Valencia), Spain
}

\begin{abstract}
An algebraic proof of the Gluing Theorem at tree level of perturbation theory in String Field Theory is given. Some applications of the theorem to closed string non-polynomial action are briefly discussed
\end{abstract}

Pacs: 11.10.-z,11.25.Sq

Key words: String Field Theory.

\footnotetext{
${ }^{1}$ Also at IFIC, Universitat de Valencia-CSIC (Centro Mixto)
} 
The gluing of vertices in String Field Theory and in particular in the case of closed strings, is at the basis of the construction of a perturbative approach to the theory. The proof of various properties such as associativity, gauge invariance of the action, etc., makes use of such gluing of vertices. To tackle the problem, Peskin, Le Clair and Preitchschopf [1] have developed and proved a theorem which shows how to construct a given vertex starting from an elementary one. In principle, one could start from the simplest vertex with three strings and generate vertices for any number of strings. With some precisions about the integration over the modular space it will give the starting point to build a field theory for closed strings with only one term, namely, the three strings vertex which appropriately will generate the infinite non-polynomial action [2].

In the proof of the theorem in [1], the authors use the Riemann surfaces with holes representation of vertices to show that the contraction of legs in different vertices (which can be represented by the BPZ inner product [3]) gives another vertex representation the Riemann surface arising from sewing two surfaces around the hole boundaries.

Moreover, it has been suggested in [4, 5] that the scattering amplitude of $\mathrm{N}$ strings, at any order in perturbation theory can be interpreted as the gluing of elementary vertices. In this approach to the field theory proposed by Witten [6], the propagator between vertices is simulated by the joining of intermediate strings in all possible parameterizations. This requires the formulation of the theory in terms of the left and right hand pieces of the string.

Following this suggestion we prove the gluing theorem using the operator approach (to string vertices) developed in [7], in which strings are expanded using left and right degrees of freedom and the Neumann functions are written in terms of infinite dimensional matrices. These matrices are just the ones appearing in the canonical transformation from the usual self-energy Fock space operators to the ones splitting left and right degrees of freedoms as mentioned above.

Using general properties of these matrices, the proof of the theorem simplifies. One can then explicitly generate the Neumann function of the Nstrings vertex starting from the 3-strings vertex appearing in the open String Field Action. In closed strings, in order to get the non-polynomial expansion, one needs to know how to contract the restricted vertex interaction [2]. Following [4] where the closed string interaction is interpreted as the 
reparametrization of the string, it appears to be possible 8 to perform a generalization of the gluing of vertices (which gives just a point in the modular space) to the gluing of parametrized vertices covering a region of the modular space.

The organization of this note is as follows. First we review the form of the vertex when the midpoint string interaction is explicitly considered. Then we briefly state the Gluing Theorem of [1]. The proof of the theorem for arbitrary vertices is sketched, and the relevant steps, in the case of the gluing of two vertices with 3 strings to give the one with four $(3+3 \rightarrow 4)$, are discussed in some detail. We will see that in the critical dimension, the ghost degrees of freedom cancel the determinant of the Laplacian coming from the gluing procedure, represented here as the determinant of an infinite dimensional matrix. Finally we state our conclusions and future outlook.

When string fields are written in terms of half string oscillator modes, the N-string vertex takes on a very elegant and simple form. In the spirit of the original work of [6] in String Field Theory, the interaction vertex is obtained by identifying oscillators referring to opposite halves of two adjacent strings. It has been shown in [9] that the Fourier components of the Neumann functions for the N-strings vertex can be written in terms of particular linear combinations of the two infinite dimensional matrices relating the half-string degrees of freedom to the conventional Hamiltonian eigenstates oscillator modes. For the purpose of this work, this particular algebraic representation of the Neumann functions is very useful; the reason is, in order to prove the theorem one needs to calculate explicitly the inverse of some combinations of these matrices which have been calculated before [9] in its most general form.

Writing the N-strings vertex in an operator form, and splitting the vertex into coordinate and ghost sector, we get for the coordinates degrees of freedom

$$
<V_{x}(1 \cdots N)\left|=\Pi_{i=1}^{N} \delta\left(\sum_{i=1}^{N} k_{i}\right){ }_{i}<k_{i}\right| V_{x}(1 \cdots N) .
$$

The $N$ momentum states ${ }_{i}<k_{i} \mid$ are eigenstates of the corresponding istring momentum operator $a_{0}^{(i)}$ and the vertex operator, written in terms of string creation and annihilation oscillator modes, is given by 


$$
V_{x}(1 \cdots N)=\exp \left\{\frac{1}{2} \sum_{r, s} \sum_{n, m} a_{\mu, n}^{(r)} N_{n, m}^{r s} a_{m}^{\mu(s)}\right\} .
$$

In $D$ space-time dimensions $\mu=1, \cdots D$ and $r, s=1, \cdots, N . N_{n, m}^{r s}$ are the Fourier components of the relevant Neumann function.

For the ghost degrees of freedom we use the bosonic formalism. Hence, one has for the ghost vertex a similar form to the matter vertex

$$
<V_{\phi}(1 \cdots N)\left|=\delta\left(\sum_{i=0} q_{i}+Q\right) \Pi_{i=1}^{N} i<-q_{i}-Q\right| V_{\phi}(1 \cdots N) .
$$

$Q$ is the weight of the curvature term in the string action [10] and the states ${ }_{i}<-q_{i}-Q \mid$ are ghost number eigenstates. In terms of the oscillator modes $\left(\phi_{n}^{(r)}\right)$ for the bosonic reparametrization ghosts one has

$$
<-q-Q\left|\phi_{0}=<-q-Q\right| q
$$

The vertex operator now takes the form

$$
\begin{aligned}
V_{\phi}(1, \cdots, N)= & \exp \left\{\frac{1}{2} \sum_{r \neq s} \phi_{0}^{(r)} \tilde{N}_{0,0}^{r, s} \phi_{0}^{(s)}+\right. \\
& \left.+\sum_{r, s} \sum_{n} \phi_{0}^{(r)} \tilde{N}_{0, n}^{r, s} \phi_{n}^{(s)}+\frac{1}{2} \sum_{r, s} \sum_{n, m} \phi_{0}^{(r)} \tilde{N}_{n, m}^{r, s} \phi_{m}^{(s)}\right\} .
\end{aligned}
$$

Notice that the ghost vertex differs from (2) in the ghost number quadratic term as well as by having an extra linear term. Coordinate and ghost coefficients are related according to

$$
\begin{aligned}
& \tilde{N}_{0,0}^{r, s}=N_{0,0}^{r, s}-\frac{1}{2} N_{0,0}^{r, r}-\frac{1}{2} N_{0,0}^{s, s}, \\
& \tilde{N}_{0, n}^{r, s}=N_{0, n}^{r, s}-\frac{1}{2} K_{n}^{s}, \\
& \tilde{N}_{n, m}^{r, s}=N_{n, m}^{r, s}
\end{aligned}
$$

where the linear coefficient represented by the vector $\vec{K}^{s}$ has as components:

$$
K_{n}^{s}=-\frac{4}{3} \frac{(-1)^{\frac{n}{2}}}{(2 n)^{\frac{1}{2}}}\left(1+(-1)^{n}\right)
$$


The discussion of both matter and ghost sectors can be carried out in parallel. The additional terms appearing in the ghost part will contribute in the critical dimension to the cancellation of the Laplacian determinant coming from the ghost and coordinate gluing. This point will be discussed later. The complete vertex is given by the product of both pieces, the one corresponding to the matter sector and that corresponding to the ghost, namely

$$
V(1, \cdots, N)=V_{x}(1, \cdots, N) V_{\phi}(1, \cdots, N) .
$$

The general form of the Neumann coefficients $N_{n, m}^{r, s}$ have been calculated in many works [11], for our purpose it is convenient the representation given in [9] since, for any vertex, they can be written in a compact form in a $\mathrm{N}$ dimensional space spanned by the $N$ strings, as elements of $N \times N$ matrix. For instance, one has for the terms which do not involve the zero modes

$$
\begin{aligned}
& N_{\text {even even }}^{r, s}=\left(\mathbf{I}_{N} M_{1}^{T}-\mathbf{S}_{+} M_{2}^{T}\right)^{-1}\left(\mathbf{I}_{\mathbf{N}} M_{2}^{T}-\mathbf{S}_{+} M_{-}^{T}\right), \\
& N_{\text {odd odd }}^{r, s}=\mathbf{S}_{+}-\mathbf{S}_{-}{ }^{T}\left(\mathbf{I}_{\mathbf{N}} M_{1}-\mathbf{S}_{+} M_{2}\right)^{-1} M_{2} \mathbf{S}_{-}, \\
& N_{\text {odd even }}^{r, s}=\mathbf{S}_{-}{ }^{T}\left(\mathbf{I}_{\mathbf{N}} M_{1}-\mathbf{S}_{+} M_{2}\right)^{-1}
\end{aligned}
$$

The remaining ones are of similar form and do not add anything new to these expressions. $\mathbf{I}_{\mathbf{N}}$ is the identity $\mathrm{N}$-dimensional matrix, whereas the matrices $\mathbf{S}_{+}$and $\mathbf{S}_{-}$are

$$
\begin{aligned}
& \left(\mathbf{S}_{+}\right)_{i j}=\frac{1}{2}\left(1+\delta_{i j}\right) \\
& \left(\mathbf{S}_{-}\right)_{i j}=\frac{(-1)^{i+1}}{2}\left(1-\delta_{i j}\right) .
\end{aligned}
$$

The infinite dimensional matrices $M_{1,2}$ give the canonical transformation from the half string degrees of freedom to the conventional ones. Explicitly they have the form [4]

$$
\begin{aligned}
& \left(M_{1}\right)_{n, m}=\frac{2}{\pi}\left(\frac{2 n}{2 m-1}\right)^{1 / 2} \frac{(-)^{n+m}}{2 n-(2 m-1)}, \\
& \left(M_{2}\right)_{n, m}=\frac{2}{\pi}\left(\frac{2 n}{2 m-1}\right)^{1 / 2} \frac{(-)^{n+m}}{2 n+2 m-1} .
\end{aligned}
$$


One can see that, after diagonalization of the $\mathbf{S}_{ \pm}$matrices, the problem of computing the vertex itself, essentially reduces to calculating the inverse of the matrix $\left(M_{1}-\cos \frac{2 k \pi}{N} M_{2}\right)$ where $k=1, \ldots, N$. Details of the whole process as well as the general form of this matrix and its inverse have been given in ref. [9].

Now, one can use this result in the proof of the Gluing Theorem [1]. The theorem states that the contraction of two string vertices (e.g., for $N$ and $N^{\prime}$ strings), through the BPZ inner product (which is nothing other than the vertex overlapping two strings, $<V(12) \mid)$, gives the $\left(N+N^{\prime}-2\right)$-string vertex. More precisely, using the notation of vertices given in equations (1) and (3), including ghost and matter sectors in the same 'bra', the result can be cast

$$
\begin{gathered}
<V(1, \ldots N-1, a)\left|<V\left(N, \ldots, N+N^{\prime}-2, a^{\prime}\right)\right| V\left(a, a^{\prime}\right)>= \\
=<V\left(1, \ldots, N+N^{\prime}-2\right) \mid .
\end{gathered}
$$

This means that we have contracted the two vertices by joining the strings $a$ and $a^{\prime}$ in the first and the second vertex respectively. Of course, because of the cyclic properties of the vertices, it is immaterial the position of these strings.

In terms of the Neumann representation of the vertex given in equations (2) and (4), the theorem shows the identification of the coefficients appearing in $V\left(1, \ldots, N+N^{\prime}-2\right)$ with particular combinations of the ones appearing in $V(1, \cdots, N-1, a)$ and $V\left(1, \cdots, N^{\prime}-1, a^{\prime}\right)$. In particular, in the proof of the theorem, and in the coordinate sector, one has to show the identifications

$$
\begin{aligned}
& \mathbf{N}^{s_{1}, s_{2}}=N^{s_{1}, s_{2}}+N^{s_{1}, a} E N^{a^{\prime}, a^{\prime}} E\left(1-N^{a, a} E N^{a^{\prime}, a^{\prime}} E\right)^{-1} N^{a, s_{2}}, \\
& \mathbf{N}^{s_{1}^{\prime}, s_{2}^{\prime}}=N^{s_{1}^{\prime}, s_{2}^{\prime}}+N^{s_{1}^{\prime}, a^{\prime}} E N^{a, a} E\left(1-N^{a^{\prime}, a^{\prime}} E N^{a, a} E\right)^{-1} N^{a^{\prime}, s_{2}^{\prime}}, \\
& \mathbf{N}^{s_{i}, s_{j}^{\prime}}=N^{s_{i}, a}-E\left(1-N^{a^{\prime}, a^{\prime}} E N^{a, a} E\right)^{-1} N^{a^{\prime}, s_{j}^{\prime}},
\end{aligned}
$$

where the indices $s_{i}\left(s_{i}^{\prime}\right)$ run from 1 to $N-1\left(N\right.$ to $\left.N^{\prime}+N-2\right)$. The boldfaced $\mathbf{N}$ represent the coefficients of the resulting glued vertex according to the index. The matrix $E_{n, m}=(-)^{(n+1)} \delta_{n, m}(n, m \geq 1)$ comes from the BPZ inner product that, in the language of vertices, is $V\left(a, a^{\prime}\right)$. Also, after the contraction of the vertices with the BPZ inner product [3], the determinant 
of the Laplacian appears. This amounts to a factor of the form

$$
\mathbf{D}_{\mathbf{x}}=\left[\operatorname{det}\left(1-N^{a^{\prime} a^{\prime}} E N^{a, a} E\right)\right]^{-D / 2},
$$

$D$ being the space time dimension, this factor will cancel once the contribution from the ghost sector is included.

Hence, the proof of the theorem relies on the calculation of the inverse matrix $\left(1-N^{a, a} E N^{a, a^{\prime}} E\right)^{-1}$. Due to the symmetries of the diagonal Neumann coefficients there is no dependence on the indices $a$ and $a^{\prime}$. The general form of this inverse matrix can be obtained in full; the algebra involved is rather tedious, and the result can be cast in terms of the matrix $M_{1}^{-1} M_{2}=\phi$. For instance, for an equal and even number of strings in each vertex, i.e. $N=N^{\prime}$ even, one can show that

$$
\begin{aligned}
& \left(1-N^{a, a} E N^{a^{\prime}, a^{\prime}} E\right)^{-1}= \\
= & \left.2 \sqrt{1-\phi^{2}}\left(1+\sqrt{1-\phi^{2}}\right) \frac{\left(1+\sqrt{1+\phi^{2}}\right)^{2 N-2}-\phi^{2 N-2}}{\left(\left(1+\sqrt{1-\phi^{2}}\right)^{N}-\phi^{N}\right.}\right)^{2} .
\end{aligned}
$$

This formula is not at all illuminating, to illustrate the essential points in the proof, we will work out in detail the particular case of the gluing of two 3 -string vertices to give the 4 -string vertex. This vertex also appears explicitly in the String Field Theory Action [6] for open strings and, as it was commented in the introduction, there is a possibility for generating the non-polynomial string field theory action using only this vertex.

More precisely we are interested in the relation

$$
<V(1,2, a)\left|<V\left(a^{\prime}, 3,4\right)\right| V\left(a, a^{\prime}\right)>=<V(1,2,3,4) \mid,
$$

which, in the momentum basis, and restricting ourselves to the coordinates degrees of freedom is written as:

$$
\begin{aligned}
& \int \Pi_{i=1}^{4} d k_{i} d k_{a} d k_{a^{\prime}} \delta\left(k_{a}-k_{1}-k_{2}\right) \delta\left(k_{a^{\prime}}-k_{3}-k_{4}\right) \delta\left(k_{a}-k_{a^{\prime}}\right) \\
& <k_{1}\left|<k_{2}\right|<k_{a}\left|V_{x}(1,2, a)<k_{a^{\prime}}\right|<k_{3}\left|<k_{4}\right| V_{x}\left(a^{\prime}, 3,4\right) V_{x}\left(a, a^{\prime}\right)\left|k_{a^{\prime}}>\right| k_{a^{\prime}}> \\
& =\int \Pi_{i=1}^{4} d k_{i} \delta\left(\sum_{i=1}^{4} k_{i}\right)<k_{1}\left|<k_{2}\right|<k_{3}\left|<k_{4}\right| V_{x}(1,2,3,4) .
\end{aligned}
$$

We focus our attention on the nonzero modes. In the coordinate sector, when zero indices are involved, no difficulties arise and the proof can be 
carried out along the same lines, the additional linear term appearing in the ghost vertex will be considered below. For the $N=3$ vertex, one has for the nonzero modes:

$$
\begin{aligned}
& N_{\text {even, even }}^{r, r}=\frac{1}{2}\left(M_{1}^{T}+\frac{1}{2} M_{2}^{T}\right)^{-1} M_{2}^{T}, \\
& N_{\text {even, even }}^{r, r \pm 1}=N_{\text {even, even }}^{r, r \pm 2}=-\frac{1}{2}\left(M_{1}^{T}+\frac{1}{2} M_{2}^{T}\right)^{-1}\left(M_{1}^{T}+M_{2}^{T}\right), \\
& N_{\text {odd, odd }}^{r, r}=\frac{1}{2}\left(M_{1}+\frac{1}{2} M_{2}\right)^{-1} M_{2}, \\
& N_{\text {odd, odd }}^{r, r \pm 1}=N_{\text {odd, odd }}^{r, r \pm 2}=-\frac{1}{2}\left(M_{1}+\frac{1}{2} M_{2}\right)^{-1}\left(M_{1}+M_{2}\right), \\
& N_{\text {even, odd }}^{r, r}=N_{\text {odd, even }}^{r, r}=0, \\
& N_{\text {even, odd }}^{r, r \pm 1}=N_{\text {odd, even }}^{r, r \pm 1}=-N_{\text {even, odd }}^{r, r \pm 2}=N_{\text {odd, even }}^{r, r \pm 2}=\left(M_{1}^{T}+\frac{1}{2} M_{2}^{T}\right)^{-1} .
\end{aligned}
$$

In this case all the information is encoded in the inverse matrix given in (9), namely

$$
M^{-1}=\left(1-N^{a^{\prime} a^{\prime}} E N^{a, a} E\right)^{-1},
$$

(notice that this matrix is effectively independent of the indices because of the symmetries of the Neumann coefficients).

To invert this matrix one needs only to make use of the properties of the matrices $M_{1,2}$ dictated by the fact that they define a canonical transformation in the string degrees of freedom [ [T]. In particular, they preserve the commutation relations of the string creation and annihilation operators, hence one has

$$
\begin{aligned}
& M_{1} M_{1}^{T}-M_{2} M_{2}^{T}=I, \\
& M_{1} M_{2}^{T}-M_{2} M_{1}^{T}=0 .
\end{aligned}
$$

A short calculation gives the inverse matrix

$$
\begin{aligned}
\left(M^{-1}\right)_{\text {even }, \text { even }} & =\left(M_{1}+\frac{1}{2} M_{2}\right)\left(1-M_{1}^{-1} M_{2}\right)\left(M_{1}^{T}+\frac{1}{2} M_{2}^{T}\right), \\
\left(M^{-1}\right)_{\text {even }, \text { odd }} & =\left(M^{-1}\right)_{\text {odd,even }}=0, \\
\left(M^{-1}\right)_{\text {odd,odd }} & =\left(M_{1}^{T}+\frac{1}{2} M_{2}^{T}\right)\left(1-M_{2} M_{1}^{-1}\right)\left(M_{1}+\frac{1}{2} M_{2}\right) .
\end{aligned}
$$


The final step is now to substitute this result in eq. (7) and complete the form of the 4-vertex. In particular one gets

$$
\begin{aligned}
\mathbf{N}^{r, r} & =\frac{1}{2}\left(\begin{array}{cc}
M_{2} M_{1}^{-1} & 0 \\
0 & -M_{1}^{-1} M_{2}
\end{array}\right) \quad, \quad \mathbf{N}^{r, r+1}=\frac{1}{2}\left(\begin{array}{cc}
-1 & \left(M_{1}^{-1}\right)^{T} \\
-M_{1}^{-1} & 1
\end{array}\right), \\
\mathbf{N}^{r, r+2} & =\frac{1}{2}\left(\begin{array}{cc}
M_{2} M_{1}^{-1} & 0 \\
0 & -M_{1}^{-1} M_{2}
\end{array}\right) \quad, \quad \mathbf{N}^{r, r+1}=\frac{1}{2}\left(\begin{array}{cc}
-1 & \left(M_{1}^{-1}\right)^{T} \\
-M_{1}^{-1} & 1
\end{array}\right),
\end{aligned}
$$

to be compared with the result given in [9] for the general vertex (we have arranged rows and columns according to the parity of the indices as in equation (11)).

Other terms relating the remaining Neumann functions involve the same sort of algebra and we will not insist on producing the calculation here. It is important to remember that, in the process of contracting the oscillators modes, the determinant term given in equation (10) appears, which will have to be combined with terms coming from the ghost sector.

Contraction of the oscillator modes in the ghost sector is performed using the same techniques. In this case, however, because of the linear factor $K_{n}^{s}$, there are additional contributions proportional to the combinations of the zero modes or, equivalently, to the total central charge $Q$.

In fact, apart from the vertex operator of the glued vertex, one obtains the above mentioned term which, combined with the determinant of the Laplacian from the coordinate sector (10) amounts to a factor

$$
\mathbf{D}_{\mathbf{x}} \mathbf{D}_{\phi} e^{\frac{1}{2} Q^{2} \mathbf{k}}
$$

$\mathbf{k}$ is given by a combination of the matrices (7) and the vector (6), whose explicit form is not needed for our purpose, and the determinant of the Laplacian, the additional piece coming from the contraction of the ghost operators using the BPZ inner product now appears to the power $\frac{-1}{2}$

$$
\mathbf{D}_{\phi}=\left[\operatorname{det}\left(1-N^{a^{\prime} a^{\prime}} E N^{a, a} E\right)\right]^{-1 / 2}
$$

To proof that this factor (13) is equal to 1 , we proceed in the following way: first consider the state generated by the moments of the energymomentum operator acting on the momentum and ghost number eigenstate

$$
<a|=<k,-q-Q| \exp \frac{1}{2}\left\{a_{n} N_{n, m}^{x} a_{m}+\phi_{n} \tilde{N}_{n, m}^{\phi} \phi_{m}\right\},
$$


(with an obvious notation for the Neumann coefficients) Due to the fact that the central charge of this operator is zero in $D=26$ space-time dimensions and $Q=-3$, we have, on the sphere, the vanishing of all moments of it. In the language of vertices this means

$$
<a\left|<a^{\prime}\right| V\left(a, a^{\prime}\right)\left|k_{a}>\right| k_{a^{\prime}}>=1 .
$$

Now, calculating explicitly the left hand side of the former relation, one arrives to the identity

$$
1=\mathbf{D}_{\mathbf{x}} \mathbf{D}_{\phi} e^{\frac{1}{2} Q^{2} \mathbf{k}}
$$

where $\mathbf{k}$ is the same contribution as the one appearing in (13), this derives from the fact that the vector $\vec{K}^{r}$ is independent of the string under consideration, namely the value of $r$, thus the $Q$ and $D$ dependence are the same in both equations. The extra coefficient in front of the glued vertex equals one thus proving the validity of the theorem.

This result completes the proof of the theorem. Other vertices can be glued following these lines, however the calculational details are more involved. This is easily seen in the form of equation (11).

Now, we want to argue that the Gluing theorem can be applied to calculate actual string amplitudes. It has been shown in [1] that string amplitudes themselves can be represented as the sum of contact string interactions in all possible reparametrization of the strings. Also in [5] it was shown that the terms of the non-polynomial closed string action can be obtained as a sum of string contact interactions, in a region of the reparametrization modular space appropriate to reproduce the so called restricted polyhedra. With this two ideas in mid, on can imagine the gluing of vertices in order to reproduce string amplitudes. For instance, starting with the three strings vertex and applying the reparametrization operator $(\Omega)$ defined in 4 to each vertex, one could envisage the construction of string amplitudes starting from a generalization of the three string vertex. More precisely one has

$$
\begin{aligned}
<V(1,2, a) \mid\left(\prod_{i=1,2, a} \Omega_{i}\right) & <V\left(a^{\prime}, 3,4\right)\left|\left(\prod_{i=a^{\prime}, 3,4} \Omega_{i}\right)\right| V\left(a, a^{\prime}\right)>= \\
& <V(1,2,3,4) \mid\left(\prod_{i=1, \ldots, 4} \Omega_{i}\right)
\end{aligned}
$$

where we have, with the BPZ inner product, glued two vertices taking all possible reparametrization of the strings to give a four string vertex which, 
in reference [4] was shown to reproduce the tree level string amplitudes for both closed and open strings.

Work in this directions is in progress and we hope to report on it in the near future. This result will open the way to simplifying the construction of amplitudes in the case of closed strings where in the non-polynomial theory of [2] requires the addition of terms in every order of perturbation theory.

To summarize, in this note we have given an algebraic proof of the Gluing Theorem of string vertices applied to the String Field Theory due to Witten [6]. We have used the representation of Neumann functions given by the half-string formulation of the theory and the problem is reduced to the calculation of inverse matrices (of infinite dimension). The introduction of the reparametrization ghosts allows us to get rid of the determinant of the Laplacian coming from the operator contraction in the process of gluing. Finally we outlook the possibility that the theorem, when applied to a suitable generalization of the string vertices, can generate the open and closed string amplitudes. This result will greatly simplify the construction of amplitudes in the context of the non-polynomial string field theory.

One of us (JB) is supported in part by grants CYCIT96-1718, PB971261 and GV98-1-80. He would also like to thank the Rutherford Appleton Laboratory for hospitality. 


\section{References}

[1] A. LeClair, M.E. Peskin and C.R. Preitschopf, Nucl. Phys. B317 (1989) 411 and Nucl. Phys. B317 (1989) 464.

[2] M. Kaku, Nucl. Phys. B267 (1986) 125.

B. Zwiebach, Ann. of Phys. 186 (1988) 111

H. Sonoda and B. Zwiebach, Nucl. Phys. B331 (1989) 592.

M. Saadi and B. Zwiebach, Ann. of Phys. 192 (1989) 213.

M. Kaku, Phys. Rev. D41 (1990) 3734.

L. Hua and M. Kaku, Phys. Lett. 250B (1990) 56.

[3] A. Belavin, A. Polyakov and A. Zamolodchikov, Nucl. Phys. B241 (1984) 333. F. Antón, A. Abdurrahman and J. Bordes, Phys. Lett. 327B (1994) 234.

[4] Chan H.M., J. Bordes, Tsou S.T. and L. Nellen, Phys. Rev. D40 (1989) 2620 and Phys. Rev. D44 (1991) 1786.

[5] A. Abdurrahman, F. Antón and J. Bordes, Phys. Lett. B327 (1994) 234.

[6] E. Witten, Nucl. Phys. B268 (1986) 253.

[7] J. Bordes, Chan Hong-Mo, L. Nellen and Tsou Sheung Tsu, Nucl. Phys. B351 (1991) 441 and A. Abdurrahman, F. Antón and J. Bordes Nucl. Phys. B397 (1993) 260.

[8] A. Abdurrahman, J. Bordes and C. Lara (work in progress).

[9] J. Bordes, A. Abdurrahman and F. Antón, Phys. Rev. D49 (1994) 2966.

[10] M. B. Green, J. H. Schwarz and E. Witten, Superstring Theory, Cambridge University Press, Cambridge 1987.

[11] A. Schwimmer, E. Gremmer and C. Thorn, Phys. Lett. 179B (1986) 57. S. Samuel, Phys. Lett. 181B (1986) 249.

A. Estaugh and J.G. McCarhy, Nuc. Phys. B294 (1987) 845.

J. Bordes and F. Lizzi, Phys. Rev. Lett. 61 (1988) 278.

D. Gross and A. Jevichi Nucl. Phys. B283 (1986) 57. 